\title{
Extracorporeal membrane oxygenation as a bridge between transfer and perioperative periods in refractory cardiogenic shock secondary to a large left atrial myxoma
}

\author{
Lijun Tian ${ }^{1}$, Suyan Zhang ${ }^{1}$, Junxian $\mathrm{Xu}^{1}$, and Xudong $\operatorname{Han}^{1}$ \\ ${ }^{1}$ Nantong University
}

November 21, 2020

\begin{abstract}
A 63-year-old man was admitted for acute left heart failure after field operations. He rapidly developed refractory cardiogenic shock due to a large left atrial myxoma which was found by bed-side echocardiography. Veno-arteriovenous extracorporeal membrane oxygenation (ECMO) was performed immediately, and the patient was transferred for further surgery with a good outcome. Therefore, timely echocardiographic evaluation and surgical removal of myxomas is recommended, and ECMO could be used as a bridge between the transfer and perioperative period.
\end{abstract}

\section{Introduction}

Cardiac myxomas are rare, and approximately $75 \%$ of them are known to develop in the left atrium. Clinical symptoms of cardiac myxomas vary from unapparent to lethal complications such as embolisms, acute heart failure, or even sudden death ${ }^{1}$. Timely diagnosis and urgent surgical treatment are crucial; however, there are a number of patients who die of severe heart failure before surgery ${ }^{2}$. Here, we report the case of a patient with a large left atrial myxoma that was asymptomatic until the acute onset of heart failure along with refractory shock. Extracorporeal membrane oxygenation (ECMO) was used as a bridge between his transfer and surgery.

\section{Case Presentation}

A 63-year-old male patient was admitted to the local hospital for acute left heart failure after field operations. He suffered from severe hypoxemia along with cardiogenic shock. He was intubated 12 hours after treatment with diuresis, cardiotonic, vasodilator, and ventilation. When our team arrived on day 2 , his blood pressure was maintained at $80 / 50 \mathrm{mmHg}$ with high-dose inotropic support (noradrenaline [100 ug/min], pituitrin $[2 \mathrm{U} / \mathrm{h}]$, and dobutamine $[20 \mathrm{ug} / \mathrm{kg} \cdot \mathrm{min}])$, and his $\mathrm{PaO} 2 / \mathrm{FiO} 2(\mathrm{P} / \mathrm{F})$ ratio was only $80 \mathrm{mmHg}$. It is worth noting that his blood pressure considerably fluctuated with heart rate. A bed-side echocardiography showed a giant anomalous vegetation bearing a strong resemblance to the evil eye (Figure. 1). He was put on venoarteriovenous extracorporeal membrane oxygenation (VAV-ECMO) for progressive cardiopulmonary failure and was transferred to our intensive care unit (ICU) 4 hours later.

On day 3 , surgery was performed through the right atrium and atrial septum, and revealed a $75 \times 30 \mathrm{~mm}$ mass attached by a narrow stalk $(10 \mathrm{~mm}$ ) originating from the atrial septum (Figure. 2). ECMO was suspended for 60 minutes during cardiopulmonary bypass and was removed 2 days later. On day 10, the patient was discharged from the ICU in good condition and was doing well on outpatient follow-up.

\section{Discussion}


The cardiovascular manifestations of patients with left atrial myxoma strongly depend on tumor size and localization $^{2}$. The patient in the present case showed respiratory failure and acute pulmonary edema as the initial signs, which were probably caused by an acute enlargement of the myxoma due to an internal hemorrhage. Unfortunately, the diagnosis of myxoma was overlooked during the initial evaluation with bedside ultrasound. The probable reason is that emergency physicians have less training in ultrasonographic image acquisition and interpretation compared with sonographers or skilled physicians that are trained in echocardiography. In addition, it is challenging to differentiate myxomas from valve vegetations and left atrial clots under severe tachycardia. Therefore, it is important to consider cardiac myxoma in the differential diagnosis of respiratory failure and acute pulmonary edema in emergency departments, especially for those patients without a history of heart disease, as early consultation with experienced sonologists.

Despite early positive internal medicine treatment, the patient's condition rapidly progressed to cardiopulmonary failure. At this time, it was discovered that the giant atrial myxoma was the cause and had to be operated immediately; however, the local hospital was unable to carry out the operation. As a result, VAV-ECMO was performed as an alternative intervention. ECMO is a rescue therapy that can stabilize patients with severe hypotension, with or without respiratory failure. In cardiology, the main indications for ECMO include cardiac arrest, cardiogenic shock, post-cardiotomy shock, refractory ventricular tachycardia, and acute management of complications of invasive procedures ${ }^{3}$. The use of ECMO as a bridge between the transfer and perioperative periods of patients with acute heart failure in cardiac surgery has been shown to be a viable strategy in the recent years; however, it is rarely reported for surgical treatment of atrial myxoma ${ }^{4}$. Therefore, the case suggests that atrial myxoma is prone to sudden death, and once diagnosed, it should be operated as soon as possible under the guidance of ECMO if necessary.

\section{Conclusion}

This case illustrates that refractory cardiogenic shock could be the first clinical manifestation of a large left atrial myxoma. Therefore, timely echocardiographic evaluation and surgical removal of the myxoma is recommended, and ECMO could be used as a bridge between the transfer and perioperative periods.

\section{Conflict of Interest}

There are no conflicts of interest to declare.

\section{Author Contributions}

Lijun Tian drafted the manuscript and conceived the study. Suyan zhang and Junxian Xu collected the patient's clinical data. Xudong Han designed the report and revised the paper. All authors read and approved the final manuscript.

\section{Data Availability Statement}

Please contact the author for data requests.

\section{Consent For Publication}

Written informed consent of clinical detail and image publication was obtained from the patient.

\section{Funding}

This work was supported by the Nantong Science and Technology Bureau [grant number, XG202003-3]; Nantong Municipal Commission of Health and Family Planning [grant numbers, MA2020016, MA2020017 and MA2020036].

\section{References}

[1] Ali MU, Finkel J. Atrial Myxoma. N Engl J Med . 2018;379(16):e26.

[2] Layton S, Ripley DP, Bellenger NG. Left atrial myxoma.BMJ . 2013;347:f4430. 
[3] Guglin M, Zucker MJ, Bazan VM, et al. Venoarterial ECMO for Adults: JACC Scientific Expert Panel. J Am Coll Cardiol . 2019;73(6):698-716.

[4] Deng L, Xia Q, Chi C, Hu G. Awake veno-arterial extracorporeal membrane oxygenation in patients with perioperative period acute heart failure in cardiac surgery. J Thorac Dis . 2020:12(5):2179-2187.

\section{Figure Legends}

Figure 1. Echocardiogram revealing "an evil eye" floating in the left atrium.(A)Systolic,(B)Diastole

Figure 2. (A)Surgical specimen of the mass (diameter: $75 \mathrm{~mm}$ ),(B)Hematoxylin and eosin staining
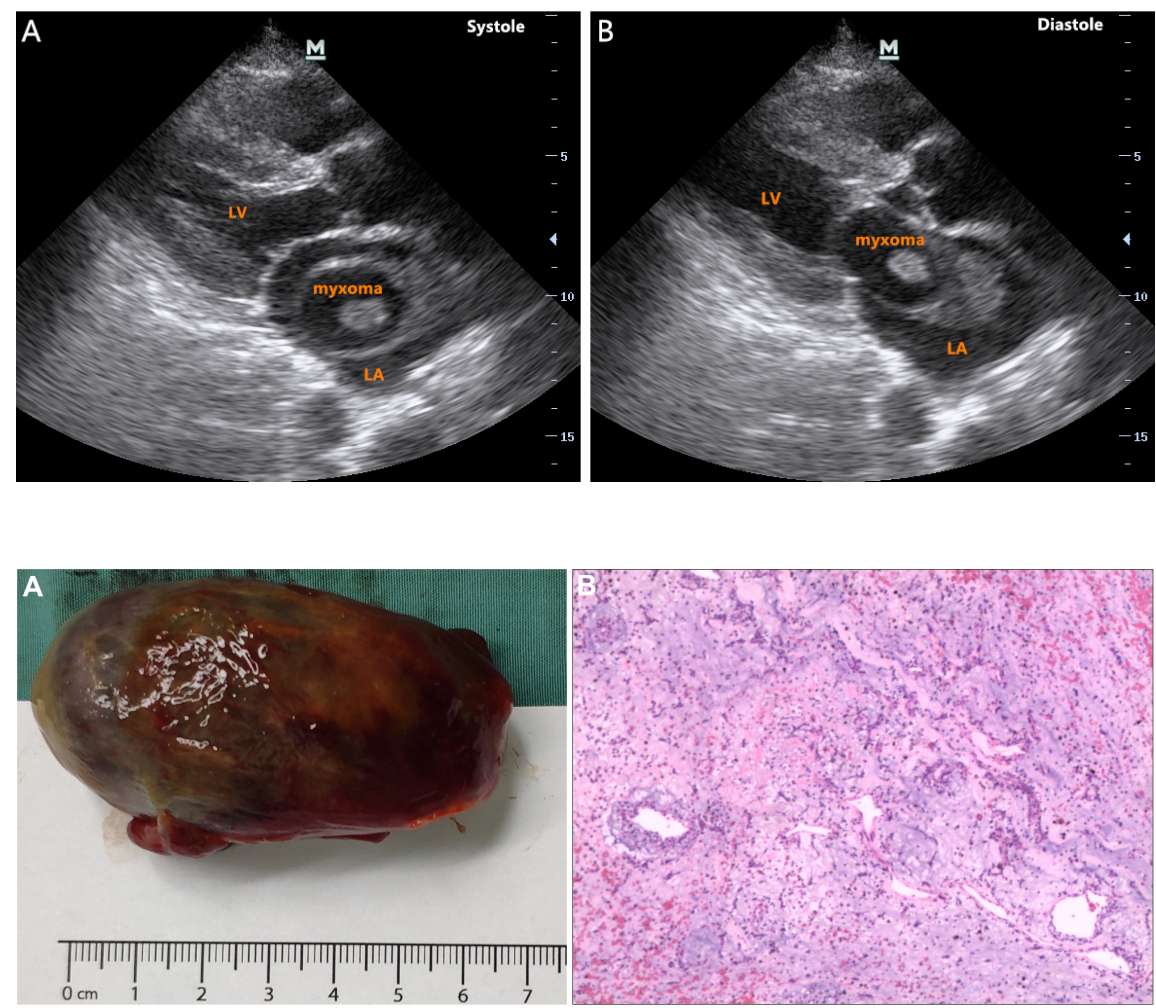